\title{
CONTROL OF A BRACHIATION ROBOT \\ WITH A SINGLE UNDERACTUATED JOINT USING NONLINEAR MODEL PREDICTIVE CONTROL
}

\author{
Vincius Menezes de Oliveira ${ }^{* 1}$ \\ Walter Fetter Lages ${ }^{* *, 2}$ \\ * Department of Mathematics, FURG, Rio Grande, Brazil \\ ** Department of Electrical Engineering, UFRGS, Porto \\ Alegre, Brazil
}

\begin{abstract}
In this paper we present the control of an underactuated brachiation robot with only one unactuated joint using the nonlinear model based predictive approach for the motion control. The robot used has 3 links, i. e., two arms and a body. We adopt the MPC to control the underactuated brachiation robot due to its advantages, mainly the construction of an optimal stabilizing control law and due to its ability to consider, in a direct way, the constraints into the optimization problem. At the end we present computational simulations with their respective results and we highlight some important considerations. Copyright (c)IFAC 2007
\end{abstract}

Keywords: Underactuated nonlinear systems, brachiation robot, NMPC

\section{INTRODUCTION}

Along the past decades the worldwide use of robotic systems has increased in a large variety of automatized activities, with a conservative forecast that points to about 1 million of robots working around the world at the end of this decade (UNECE 2004). The most robotized economic activity is the automotive industry, where the assembly and welding tasks are executed by robotic manipulators.

There are various practical situations where one can use service robots, for example, gas station (refueling of vehicles), agriculture and forestry, construction industry, renovation of nuclear power plant, hobbies and recreation. Moreover, one activity that is getting attention from researchers and robot manufacturers is the inspection task.
Robots for inspection are already used in various sectors, such as nuclear power plants and pipes (electrical and gas).

One of the inspection tasks that are on top of research is the inspection of power transmission lines, where the traditional methods of inspection involve tedious and high cost procedures. Visual inspection executed by human operators over long extension of cables and a high number of isolators is undoubtedly a problem. Due to the repetitive scene of cables and isolators the inspection of such elements is monotonous and the quality of the inspection result cannot be guaranteed. Many papers in the literature have considered the application of service robots for this task, but it is still an open subject for investigation (Côté et al. 2000, Aracil et al. 2002, Campos et al. 2003, de Souza et al. 2004, Rocha and ao Siqueira 2004).

\footnotetext{
1 Email: vinicius@ieee.org

2 Email: w.fetter@ieee.org
} 
Considering the inspection of power transmission lines, robots are already used in underground cable systems. Recently, in New York city, a robot to inspect underground cables was commissioned. This work proposes the development of a robot for the inspection of overhead ground power transmission lines, instead of underground ones. The inspection of overhead wires implies a suitable locomotion system to move along the line and to avoid the fall of the robot. Hence, effective locomotion systems to overcome obstacles, such as aircraft warning spheres, counterweights and towers are needed.

In the beginning of the past decade a new class of mobile robot was proposed by Prof. Toshio Fukuda in (Fukuda et al. 1991b), presenting a sixlink full-actuated brachiation robot that imitates the movement of an ape swinging from branch to branch. This robot makes effective use of gravity for swinging and to move itself. In the sequel a simpler two-link brachiation robot was proposed (Fukuda et al. 1991a), where a control system using CMAC (Cerebellar Model Arithmetic Computer) was developed, considering heuristic creation of input driving forces in a previous training. Moreover, this two-link robot was designed as an underactuated system, i. e., the robot has more degrees of freedom than control inputs. The control of underactuated mechanical systems is a research topic that during the past years has been in vogue in the literature (Oriolo and Nakamura 1991, Reyhanoglu et al. 1996, Su and Stepanenko 1999, Nakanishi et al. 1999).

The study of brachiation systems, specifically the underactuated ones, can be justified by situations where it is necessary to reduce the weight of the robot or when it is desired to maximize the time of autonomy of the robot (minimizing the energy consumption). Moreover, from the point of view of the control theory, underactuated mechanical systems present second order non-integrable differential constraints, commonly referred to as nonholonomic second order systems (Luca and Iannitti 2002). The control of such systems has attracted the attention of the community for a long time due to the impossibility of stabilizing it around an equilibrium point via smooth timeinvariant feedback control.

The objective of this work is to consider the motion control of a 1-underactuated brachiation robot, i. e., a robot with one degree of freedom that is not directly controlled. The control architecture used is the NMPC scheme because it can deal with constraints on inputs and state and generates an implicit optimal control law (de Oliveira 1996). Bearing in mind the idea of take advantage of gravity forces to move the robot, it can be supposed that the optimal control would be one that makes effective use of such forces. Hence, the NPMC would generate a control that exploit the gravity forces.

This paper is organized as follows. In section 2 is presented the dynamics of the brachiation robot and in section 3 the nonlinear model-based predictive control scheme is presented. In the sequel, in section 4 we give some results from two different initial positions. Concluding, some discussion concerning the performance is carried out. We give some directions for further development.

\section{DYNAMICAL MODELING}

Considering the brachiation robot (seen in figure 1) as a serial open-chain robotic manipulator, its dynamics can be generally given by:

$$
M(\theta) \ddot{\theta}(t)+V(\theta, \dot{\theta}) \dot{\theta}(t)+G(\theta)=B \tau-F_{v}(\dot{\theta})
$$

where $\theta=\left[\begin{array}{lll}\theta_{1} & \theta_{2} & \theta_{3}\end{array}\right]^{T}$ is the vector with the joints coordinates, $M(\theta) \in \mathcal{R}^{3 \times 3}$ is a symmetric matrix representing the inertial terms, $V(\theta, \dot{\theta}) \in \mathcal{R}^{3 \times 3}$ is a matrix with the Coriolis and centrifugal terms, $G(\theta) \in \mathcal{R}^{3 \times 1}$ is a vector with the gravity force terms and $F_{v} \in \mathcal{R}^{3 \times 1}$ represents the viscous friction terms.

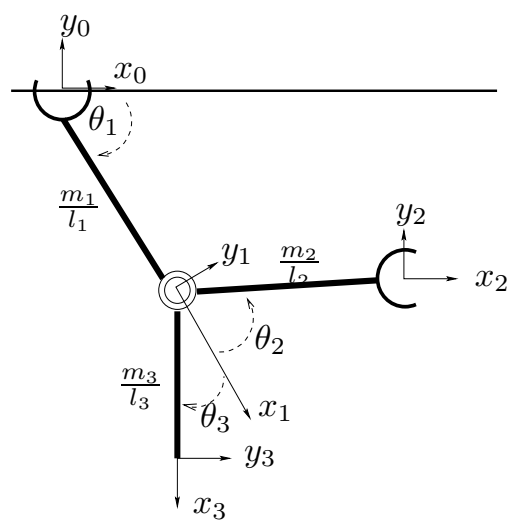

Fig. 1. Underactuated brachiation robot.

The robot used in this work is designed as an underactuated system, i. e., a system in which the number of control inputs $m$ is smaller than the number of degrees of freedom $n(m<n)$. In other words, the dimension of the input vector $\tau \in \mathcal{R}^{m \times 1}$ is smaller than the dimension of the state vector $\theta \in \mathcal{R}^{n \times 1}$. The input selection matrix $B$ is a real matrix of dimension $(n \times m)$.

The equations of the dynamics of the robot given by (1) can be rewritten in a more suitable state space form:

$$
\dot{q}(t)=f(q)+g(q) u
$$

where $q=\left[\begin{array}{ll}\theta & \dot{\theta}\end{array}\right]^{T}$ is a $6 \times 1$ vector of generalized coordinates, $u=B \tau$ and

$$
f(q)=\left[\begin{array}{c}
\dot{\theta} \\
-M^{-1}(q)\left[V(q) \dot{\theta}+G(q)+F_{v}(q)\right]
\end{array}\right]
$$


and

$$
g(q)=\left[\begin{array}{c}
0_{3 \times 3} \\
M^{-1}(q)
\end{array}\right]
$$

In this paper we have considered that the system has $m=2$ input controls, applied on joint 2 and on joint 3 , as depicted in figure 2 , defined as:

$$
\tau=\left[\begin{array}{l}
\tau_{2} \\
\tau_{3}
\end{array}\right]
$$

and the input selection matrix $B$ is given by:

$$
B=\left[\begin{array}{ll}
0 & 0 \\
1 & 0 \\
0 & 1
\end{array}\right]
$$

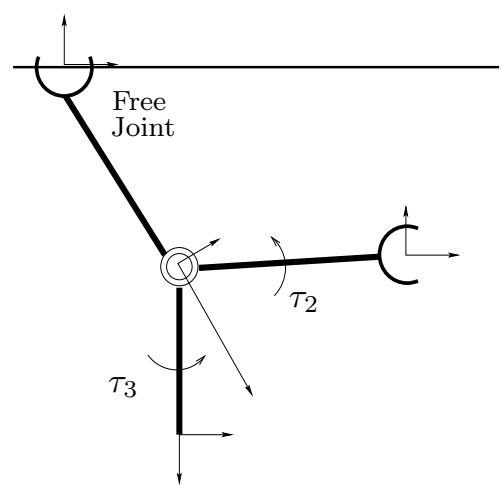

Fig. 2. Actuated and underactuated joints.

\section{MPC SCHEME}

In the following we will develop the the NMPC scheme proposed in this work, represented by the block diagram in figure 3 .

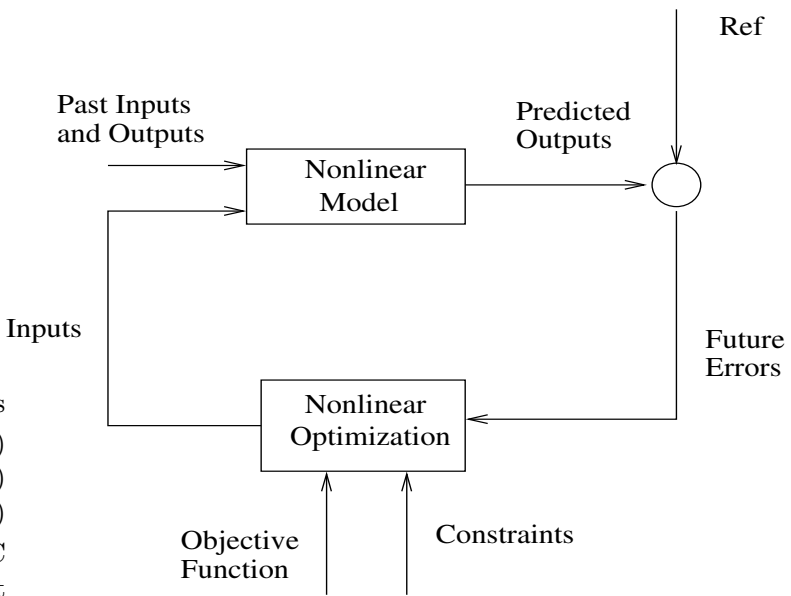

Fig. 3. General NMPC scheme.

The basic elements of the model-based predictive controller are: nonlinear prediction model, nonlinear optimization procedure and objective function. The model is used to predict the future plant outputs, based on past and current values and on the proposed optimal future control actions. The optimization procedure is responsible of calculating the optimal future control, taking into account the objective function, as well as the constraints (Camacho and Bordons 1999). The objective function defines the criteria to be optimized (usually involving the future errors) in order to force the generation of a control sequence that drives the system as desired.

Now, consider a general nonlinear model, expressed as:

$$
\dot{x}(t)=f(x(t), u(t))
$$

where $x(t)$ is the state vector and $u(t)$ is the control input vector. The nonlinear model, now described in discrete time, is given by:

$$
x(k+1)=f(x(k), u(k))
$$

The objective function to be minimized assumes, in general, the following form:

$$
\begin{aligned}
\Phi(t) & =\sum_{j=1}^{N} x^{T}(k+j \mid k) \mathbf{Q} x(k+j \mid k) \\
& +\sum_{j=1}^{N} u^{T}(k+j-1 \mid k) \mathbf{R} u(k+j-1 \mid k)
\end{aligned}
$$

where $N$ is the prediction and control horizon and $\mathbf{Q} \geq 0$ and $\mathbf{R} \geq 0$ are weighting matrices that penalize the state error and the control effort, respectively.

Considering the fact that every real system is in practice subjected to some constraint (for example physical limits), we define the following general constraint expressions:

$$
\begin{array}{ll}
x(k+j \mid k) \in \mathcal{X}, & j \in[1, N] \\
u(k+j \mid k) \in \mathcal{U}, & j \in[0, N]
\end{array}
$$

where $\mathcal{X}$ is the set of all possible values for $x$ and $\mathcal{U}$ is the set for all possible values for $u$. By supposing that such constraints are linear with respect to $x$ and $u$, we can write:

$$
\begin{array}{ll}
\mathbf{C} x(k+j \mid k) \leq c, & j \in[1, N] \\
\mathbf{D} u(k+j \mid k) \leq d, & j \in[0, N]
\end{array}
$$

Thus, the optimization problem, to be solved at each sample time $k$, is to find a control sequence $u^{\star}$ and a state sequence $x^{\star}$ such that minimize the objective function $\Phi(k)$ under imposed constraints, that is:

$$
u^{*}, x^{*}=\arg \min _{u, x}\{\Phi(k)\}
$$

subjected to: 


$$
\begin{array}{rlrl}
x(k \mid k) & =x_{0} & & (11) \\
x(k+j \mid k) & =f(x(k+j-1 \mid k), & u(k+j-1 \mid k)), \\
C x(k+j \mid k) & \leq c, & j \in[1, N] & (12) \\
D u(k+j \mid k) & \leq d, & j \in[1, N] &
\end{array}
$$

where $x_{0}$ is the value of $x$ in instant $k$.

The problem of minimizing (10) is solved for each sampling time, resulting in the optimal control sequence:

$$
u^{*}=\left\{u^{*}(k \mid k), u^{*}(k+1 \mid k), \ldots, u^{*}(k+N \mid k)\right\}
$$

and the optimal state sequence is given by:

$$
x^{*}=\left\{x^{*}(k+1 \mid k), \ldots, x^{*}(k+N \mid k)\right\}
$$

with an optimal cost $\Phi^{*}(k)$. Thus, the control law defined by NMPC is implicitly given by the first term of the optimal control sequence:

$$
h(\delta)=u^{*}(k \mid k)
$$

where $h(\delta)$ is a continuous variable held constant for the sampling interval $T$. Hence, from the above, the closed-loop system reads:

$$
\dot{x}(\delta)=f(x(\delta), h(\delta))
$$

\subsection{Brachiation Robot Control}

According to the equations of the proposed NMPC, it is necessary to describe the dynamics of the system in discrete time. Thus, the system given by (2) can be discretized by using the Euler's method and one can rewrite:

$$
q(k+1)=q(k)+T f(k)+T g(k) u(k)
$$

where $T$ is the sampling interval.

The objective function takes into account the cartesian position and velocity of the end-effector of the robot, instead of considering directly the joint coordinates, because the robot must reach the supporting line $(y=0)$ with null velocity, independently of the joint configuration. It is important to highlight the fact the robot is not fully actuated. Thus, the objective function is given by:

$$
\begin{aligned}
\Phi(t) & =\sum_{j=1}^{N} X^{T}(k+j \mid k) \mathbf{Q} X(k+j \mid k) \\
& +\sum_{j=1}^{N} \dot{X}^{T}(k+j \mid k) \mathbf{R} \dot{X}(k+j \mid k) \\
& +\sum_{j=1}^{N} u^{T}(k+j-1 \mid k) \mathbf{S} u(k+j-1 \mid k)(20)
\end{aligned}
$$

where $X=\left[\begin{array}{ll}x & y\end{array}\right]^{T}$ is the cartesian coordinates vector, $\dot{X}=\left[\begin{array}{ll}\dot{x} & \dot{y}\end{array}\right]^{T}$ is the cartesian velocities vector. The matrices $Q, R$ and $S$ are of dimension $2 \times 2$.

The NMPC deals with the system constraints as described by equations (8) and (9): $D=$ $[1-1]^{T}, d=\left[\tau_{\max } \tau_{\max }\right]^{T}, C=[I-I]^{T}$ and $c=\left[\theta_{\max } \theta_{\max }\right]^{T}$, where $\tau_{\max }$ is maximum torque and $\theta_{\max }=\pi$ is the maximum angular displacement of each joint.

\section{RESULTS}

In this section we present the results obtained to the control of an 1-underactuated brachiation robot using NMPC. We have considered 2 different initial positions for the robot: the first one with the two arms horizontally stretched $\left(q_{0}=\right.$

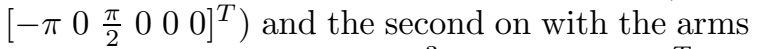

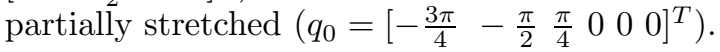

The simulation was implemented using the software Matlab ${ }^{\circledR}$, with 500 iterations and a sampling time of $10 \mathrm{~ms}$ and a control interval of $20 \mathrm{~ms}$. The dynamics parameters of the robot used in the simulations are shown in table 1.

Table 1. Parameters of the robot.

\begin{tabular}{|c|c|c|c|c|}
\hline Parameter & Unit & Joint 1 & Joint 2 & Joint 3 \\
\hline \hline Mass & $\mathrm{Kg}$ & 1.5 & 1.5 & 2.0 \\
\hline Length & $\mathrm{m}$ & 1.0 & 1.0 & 1.5 \\
\hline Viscous Friction & $\mathrm{Nm} / \mathrm{s}$ & 0.1 & 0.1 & 0.4 \\
\hline \hline
\end{tabular}

For both simulations, the parameters of the controller used are:

- prediction horizon $N=5$;

- $Q=\operatorname{diag}(5,15)$;

- $R=\operatorname{diag}(0.01,0.01)$;

- $S=0.01$;

and the constraints values are:

- maximum joint displacement $q_{\max }=\pi(r d)$;

- maximum joint velocity $\dot{q}=20(\mathrm{rd} / \mathrm{s})$

- maximum torque $\tau_{\max }=30(\mathrm{Nm})$.

Figure 4 shows the angular position of each joint, for the horizontally stretched configuration and in figure 5 one can observe the joints position for the partially stretched initial position.

The external torques applied on joint 2 and on joint 3 for both simulations can be observed, respectively, in figures 6 and 7. In figure 8 we have the values of the objective function for the two simulations.

The end-effector trajectory of the robot for the first simulation is in figure 9. and for the second one is in figure 10 .

Considering the horizontal displacement of the end effector in both simulations, the robot moves 


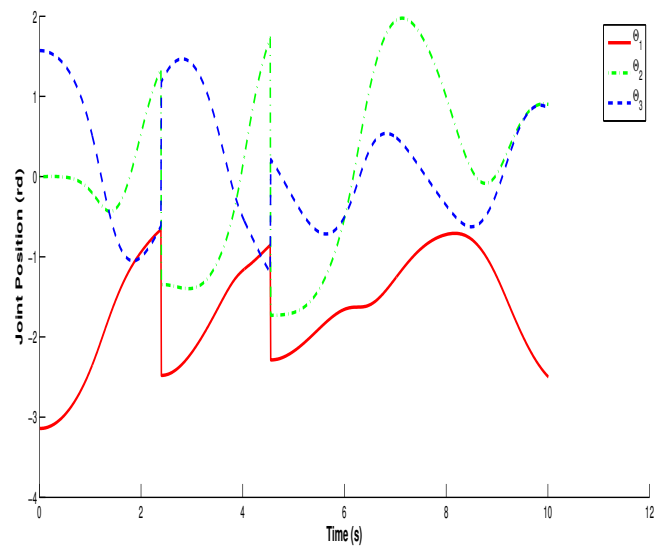

Fig. 4. Joint coordinates for simulation 1.

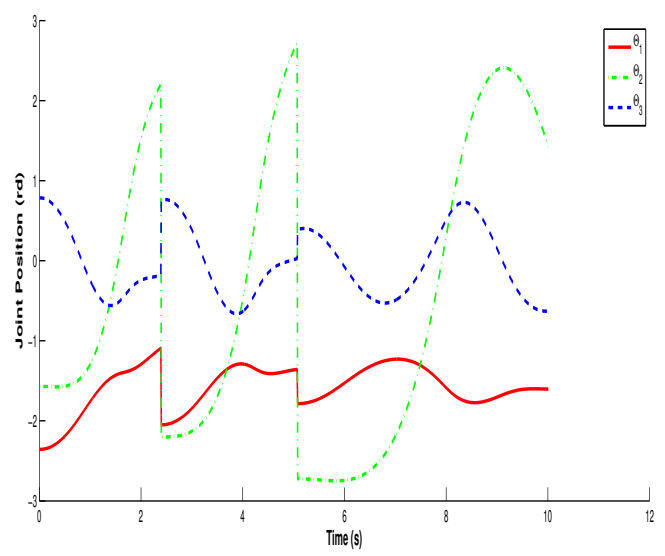

Fig. 5. Joint coordinates for simulation 2.

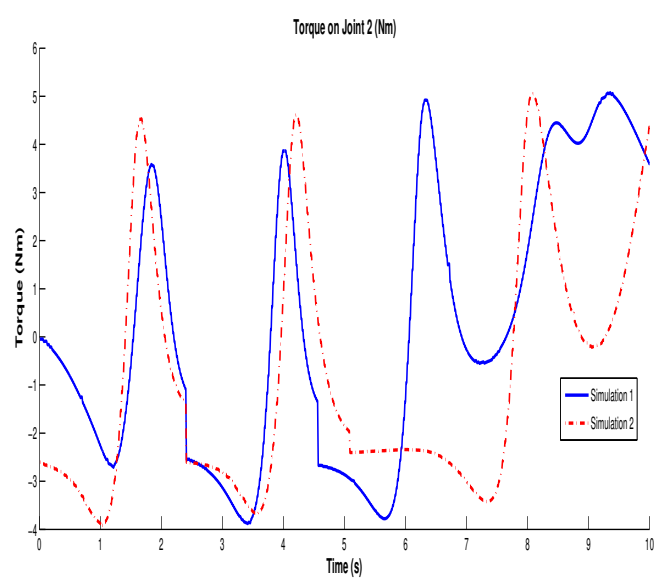

Fig. 6. Torques applied on joint 2 .

far from the origin when horizontally stretched than partially stretched. Once the torques applied are very similar (a bit smaller for the horizontal configuration), we conclude that it is caused by the gravity force.

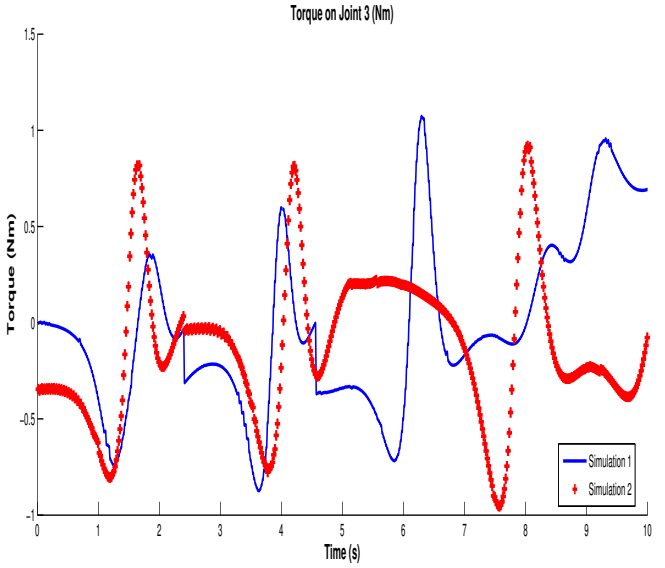

Fig. 7. Torques applied on joint 3 .

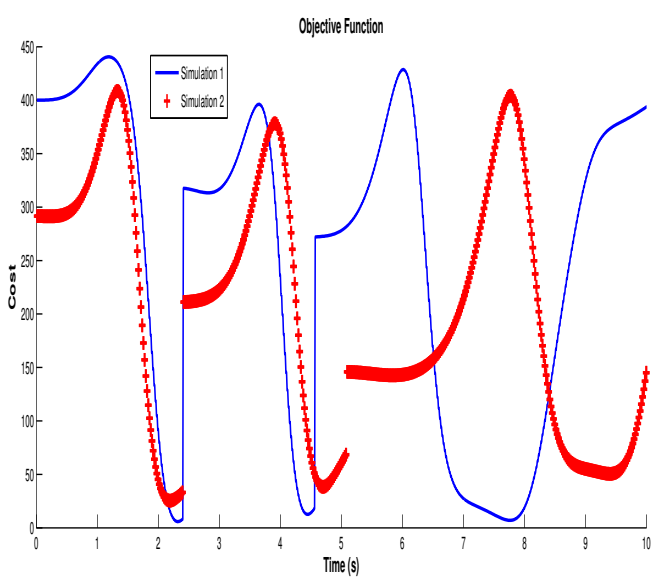

Fig. 8. Objective function values.

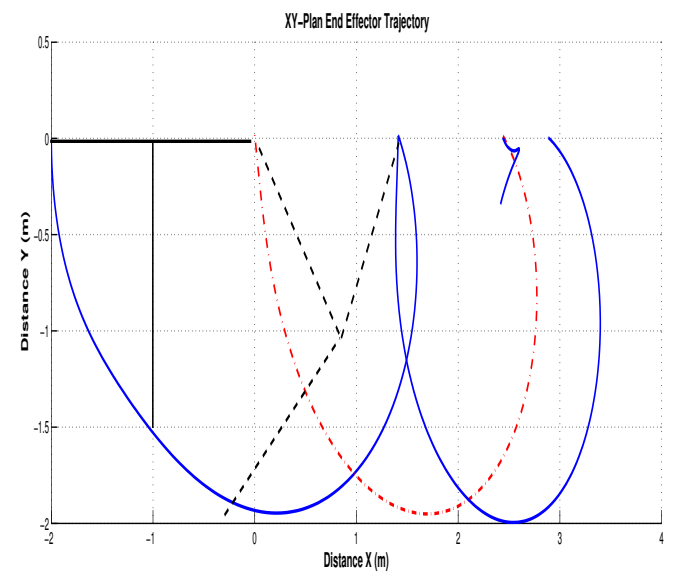

Fig. 9. Cartesian position of the end effector for simulation 1.

\section{CONCLUSIONS}

In this work we have addressed the control of an 1-underactuated brachiation robot using the nonlinear model based predictive approach. Once the system is underactuated, we have to consider its constrained part to calculate the necessary control action to move the robot. This is one of the reasons why we have adopted the NMPC strategy 


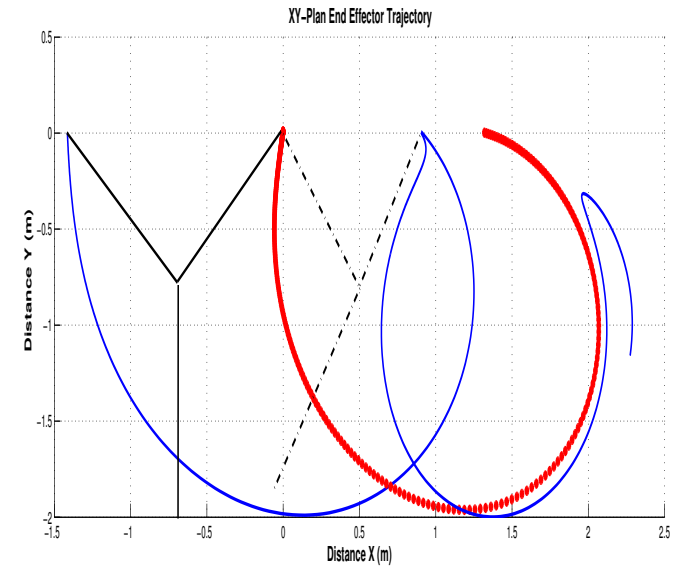

Fig. 10. Cartesian position of the end effector for simulation 2 .

of control. We observed that the controller was able to control the brachiation motion of the robot along the horizontal line, dealing with the nonholonomic constraint and respecting the limits imposed (torque, angular displacement, angular velocity). The next steps are to perform a formal analysis of the system stability and to implement the proposed controller in real time.

\section{ACKNOWLEDGMENTS}

The authors gratefully acknowledge the financial support from CAPES (Coordenação de Aperfeiçoamento de Pessoal de Nível Superior) and from CEEE (Companhia Estadual de Energia Elétrica).

\section{REFERENCES}

Aracil, R., M. Ferre, M. Hernando, E. Pinto and J. M. Sebastian (2002). Telerobotic system for live-power line maintenance. Control Engineering Practice 10(11), 1271-1281.

Camacho, Eduardo F. and Carlos Bordons (1999). Model Predictive Control. Advanced Textbooks in Control and Signal Processing. Springer-Verlag.

Campos, Mário F. M., Guilherme A. S. Pereira, Samuel R. C. Vale, Alexandre Q. Bracarence, Gustavo A. Pinheiro and Maurício P. Oliveira (2003). A robot for installation and removal of aircraft warning spheres on aerial power transmission lines. IEEE Transactions on Power Delivery 18(4), 1581-1582.

Côté, Jacques, Serge Montambault and MIchel St.-Loius (2000). Preliminary results on the development of a teleoperated compact trolley for live-line maintenance. In: IEEE Conference on Transmission and Distribution Construction, Operation and Live-line Maintenance. pp. 21-27. de Oliveira, Simone Loureiro (1996). Model Predictive Control for Constrained Nonlinear Systems. PhD thesis. California Institue of Technology (CALTECH). California - USA.

de Souza, Adinan, Lucas Antonio Moscato, Melquisedec Francisco dos Santos, Walter de Brito Vidal Filho, Gustavo André Nunes Ferreira and Armindo Gustavo Ventrella (2004). Inspection robot for high-voltage transmission lines. In: ABCM Symposium Series in Mechatronics (ABCM BrazilianSociety of Mechanical Sciences and Engineering, Eds.). Vol. 1.

Fukuda, Toshio, Fuminori Saito and Fumihito Arai (1991a). A study on the brachiation type of mobile robots (heuristic creation of driving input and control using cmac). In: IEEE/RSJ International Workshop on Intelligent Robots and Systems. Vol. 2. pp. 478-483.

Fukuda, Toshio, Hidemi Hosokal and Yuji Kondo (1991b). Brachiation type of mobile robot. In: International Conference on Advanced Robotics. Vol. 2 of Robots in Unstructured Environments. pp. 915-920.

Luca, Alessandro De and Stefano Iannitti (2002). A simple stlc test for mechanical systems underactuated by one control. In: Proceedings of the IEEE International Conference on Robotics \& Automation. pp. 1735-1740.

Nakanishi, Jun, Toshio Fukuda and Daniel E. Koditschek (1999). Brachiation on a ladder with irregular intervals. In: Proceedings of the 1998 IEEE Internation Conference on Robotics and Automation. Detroit, Michigan. pp. $2717-2722$.

Oriolo, Giuseppe and Yoshihiko Nakamura (1991). Control of mechanical systems with secondorder nonholonomic constraints: Underactuated manipulators. In: Proceedings of the $30^{\text {th }}$ Conference on Decision and Control. pp. 2398-2403.

Reyhanoglu, Mahmut, Arjan van der Schaft, N. Harris McClamroch and Ilya Kolmanovsky (1996). Nonlinear control of a class of underactuated systems. In: Proceedings of the $35^{\text {th }}$ Conference on Decision and Control. pp. 1682-1687.

Rocha, José and Jo ao Siqueira (2004). New approaches for surveillance tasks. In: $5^{\text {th }}$ IFAC/EUROn SYmposium on Intelligent Autonomous Vehicles. jrocha@est.ips.pt.

$\mathrm{Su}$, Chun-Yi and Yury Stepanenko (1999). Adaptive variable structure set-point control of underactuated robots. IEEE Transactions on Automatic Control 44(11), 2090-2093.

UNECE (2004). Worldwide growth in the period 2004-2007. Technical report. United Nations Economic Commission for Europe - World Robotics. 